\title{
RESEARCH
}

Open Access

\section{Effects of host vimentin on Eimeria tenella sporozoite invasion}

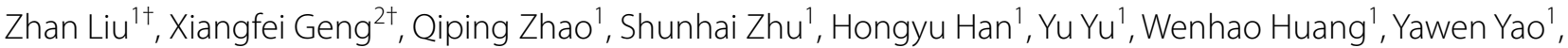 \\ Bing Huang ${ }^{1}$ and Hui Dong ${ }^{1 *}$
}

\begin{abstract}
Background: Chicken coccidiosis is a parasitic disease caused by Eimeria of Apicomplexa, which has caused great economic loss to the poultry breeding industry. Host vimentin is a key protein in the process of infection of many pathogens. In an earlier phosphorylation proteomics study, we found that the phosphorylation level of host vimentin was significantly regulated after Eimeria tenella sporozoite infection. Therefore, we explored the role of host vimentin in the invasion of host cells by sporozoites.

Methods: Chicken vimentin protein was cloned and expressed. We used qPCR, western blotting, and indirect immunofluorescence to detect levels of mRNA transcription, translation, and phosphorylation, and changes in the distribution of vimentin after E. tenella sporozoite infection. The sporozoite invasion rate in DF-1 cells treated with vimentin polyclonal antibody or with small interfering RNA (siRNA), which downregulated vimentin expression, was assessed by an in vitro invasion test.
\end{abstract}

Results: The results showed that vimentin transcription and translation levels increased continually at 6-72 $\mathrm{h}$ after $E$. tenella sporozoite infection, and the total phosphorylation levels of vimentin also changed. About $24 \mathrm{~h}$ after sporozoite infection, vimentin accumulated around sporozoites in DF-1 cells. Treating DF-1 cells with vimentin polyclonal antibody or downregulating vimentin expression by siRNA significantly improved the invasion efficiency of sporozoites.

Conclusion: In this study, we showed that vimentin played an inhibitory role during the invasion of sporozoites. These data provided a foundation for clarifying the relationship between Eimeria and the host.

Keywords: Eimeria, Invasion, Vimentin, Invasion assay

*Correspondence: donghui@shvri.ac.cn; donghui@caas.cn

${ }^{\dagger}$ Zhan Liu and Xiangfei Geng contributed equally to this work

1 Key Laboratory of Animal Parasitology of Ministry of Agriculture,

Shanghai Veterinary Research Institute, Chinese Academy of Agricultural

Sciences, Minhang 200241, Shanghai, People's Republic of China

Full list of author information is available at the end of the article permits use, sharing, adaptation, distribution and reproduction in any medium or format, as long as you give appropriate credit to the original author(s) and the source, provide a link to the Creative Commons licence, and indicate if changes were made. The images or other third party material in this article are included in the article's Creative Commons licence, unless indicated otherwise in a credit line to the material. If material is not included in the article's Creative Commons licence and your intended use is not permitted by statutory regulation or exceeds the permitted use, you will need to obtain permission directly from the copyright holder. To view a copy of this licence, visit http://creativecommons.org/licenses/by/4.0/. The Creative Commons Public Domain Dedication waiver (http://creativeco mmons.org/publicdomain/zero/1.0/) applies to the data made available in this article, unless otherwise stated in a credit line to the data. 


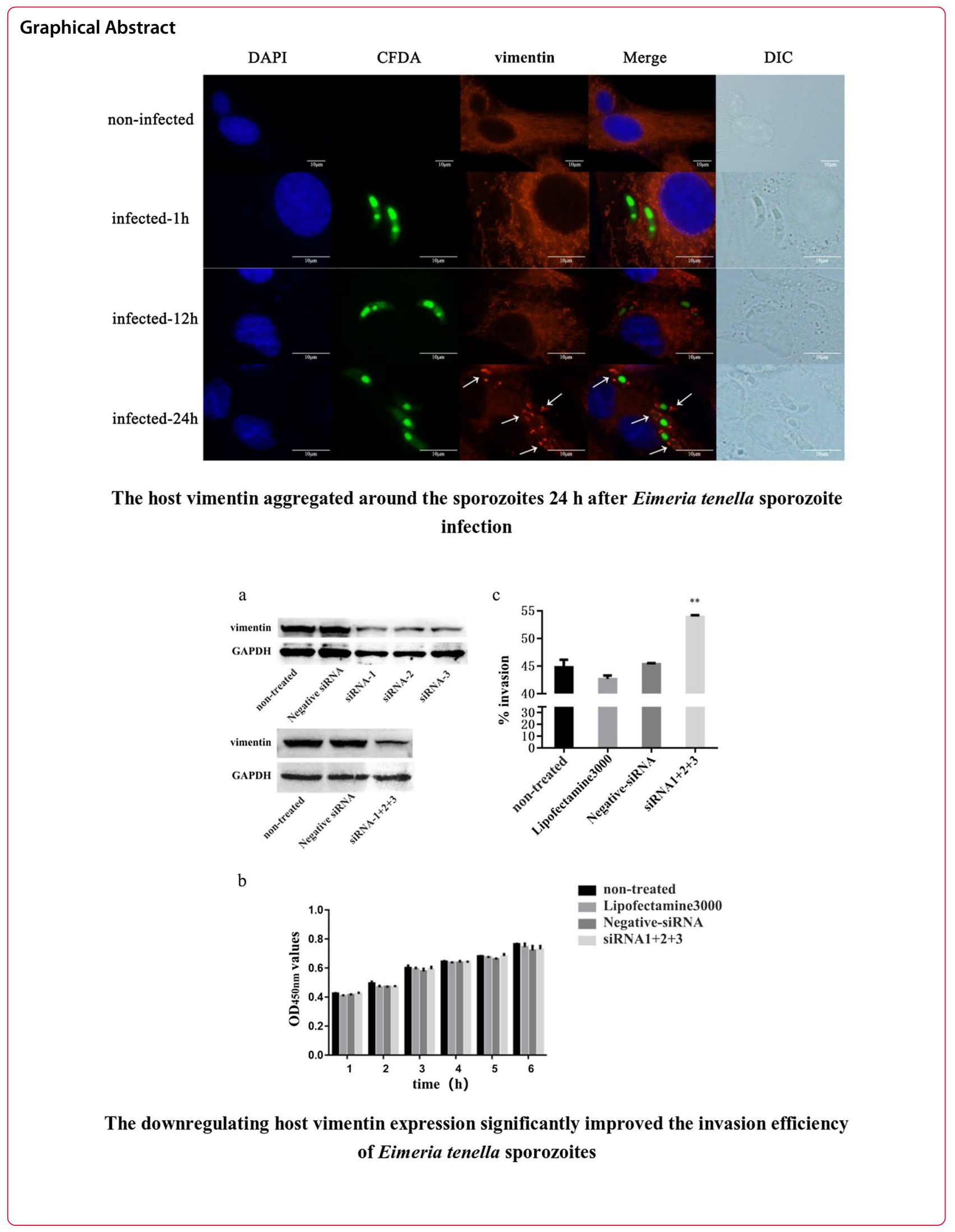




\section{Background}

Coccidiosis in chickens is caused by infection with parasites of the genus Eimeria and is one of the most common and damaging parasitic diseases in poultry farming, causing economic losses of approximately $£ 2$ billion a year worldwide [1]. Conventional methods for the prevention and control of coccidiosis include anti-coccidian drugs and live vaccines; however, these measures give rise to several issues with regard to drug resistance, food security, production cost, and the cross-protection of species [2-4]. Therefore, there is an urgent need to seek new ideas and perspectives on the prevention and control of coccidiosis.

Eimeria tenella has the strongest pathogenicity among the known chicken Eimeria species. As an obligate intracellular parasite, E. tenella must rely on host cells to complete the whole life cycle [5]. Therefore, the identification of key proteins involved in the invasion process is particularly important for exploring the molecular mechanism of coccidian infection and finding a breakthrough method to prevent and control coccidiosis [6].

The mechanism of $E$. tenella invasion in host cells has been explored for many years. Research developments have provided growing evidence that the $E$. tenella infection process is not the independent behavior of the parasite, but a complex process with the participation of host cells. For instance, invasion of host cells by E. tenella causes host F-actin-specific aggregation on the surface of sporozoites. This aggregation is closely related to the invasion efficiency of E. tenella, and the inhibition of F-actin aggregation by cytochalasin $\mathrm{D}$ will reduce the invasion rate of E. tenella [7]. Eimeria tenella sporozoites can be attached to the intestinal epithelium through the duodenal mucin of the chicken, and host proteins can significantly inhibit the invasion of the cells by sporozoites [8]. The expression of host fatty acid-binding protein 4 increased significantly after $72 \mathrm{~h}$ of invasion by $E$. tenella sporozoites, and the overexpression of this protein significantly inhibited the invasion [9]. Processing of the host cells with polyclonal antibodies against the receptor for activated $C$ kinase 1 can promote the invasion of E. tenella sporozoites [10]. These results demonstrate that host proteins play an indispensable role in $E$. tenella invasion.

In our previous study, we used tandem mass taglabeled quantitative phosphorylation proteomic technology and parallel reaction monitoring to screen and verify the significantly regulated phosphoproteins of chicken embryo fibroblast cell line (DF-1) cells infected with $E$. tenella sporozoites. The results showed that the phosphorylation level of host vimentin changed significantly after infection. The phosphorylation levels of vimentin at Ser6 and Ser7 sites were significantly higher than those of the uninfected cells $6 \mathrm{~h}$ and $36 \mathrm{~h}$ after infection by sporozoites, while the phosphorylation level at Ser 4 sites was significantly lower $36 \mathrm{~h}$ after infection than after $6 \mathrm{~h}$ (unpublished data). Host vimentin is a key protein in the process of infection of many pathogens, but its role differs among different pathogens [11-14], and its role in Eimeria infection has not been explored. Therefore, we explored the role of host vimentin in the invasion of host cells by E. tenella sporozoites.

\section{Methods}

\section{Parasites, cells, and experimental animals}

The Shanghai strain of E. tenella (CAAS21111611) used in the current study has been retained in our laboratory since 1993 and was propagated by passage through 14-day-old coccidia-free chickens [15]. Sporozoites were collected and purified from cleaned sporulated oocysts using standard procedures [16, 17]. DF-1 cells (ATCC CRL-12203) were cultured in Dulbecco's modified Eagle's medium (DMEM) (Gibco BRL, Paisley, UK) supplemented with $10 \%$ fetal bovine serum (FBS) (Gibco) and $1 \%$ penicillin/streptomycin (PS) (Gibco) at $37{ }^{\circ} \mathrm{C}$ in a $5 \%$ $\mathrm{CO}_{2}$ incubator. Chinese yellow chickens were purchased from Shanghai Fuji Biotechnology Co., Ltd., and kept in an environment without coccidia.

\section{Cloning and identification of the chicken vimentin gene}

The total RNA in DF-1 cells was extracted using a MiniBEST Universal RNA Extraction Kit (TaKaRa, Dalian, China) and the first-strand cDNA was prepared using total RNA and a reverse transcription kit (TaKaRa). The primers were designed according to the coding region (1383-base-pair [bp]) sequence of the Gallus gallus (chicken) vimentin gene (GenBank accession number: NM_001048076.2) as follows: forward primer: 5'-CGGAATTCATGAGC TTCACCAGCAGCAA-3', reverse primer: $5^{\prime}$-CCCTCGAGTTACTCCAAGTCATC GTGATGCTG-3', including EcoRI and XhoI restriction enzyme cutting site, respectively (underlined label). The chicken vimentin gene was amplified and cloned into the expression vector pGEX-4 T-1 to construct the recombinant plasmid pGEX-4 T-vimentin. Then the recombinant plasmid was transformed into Escherichia coli TOP10 competent cells (Tiangen Biotech, Beijing, China) and selected with Luria-Bertani (LB) solid medium containing ampicillin. The positive-screened colonies were sent to the Shanghai Qingke Company for sequencing. Basic Local Alignment Search Tool (BLAST) programs at the National Center for Biotechnology Information (http:// www.ncbi.nlm.nih.gov/BLAST/) were used for the similarity analysis of the sequencing results and vimentin gene full-length sequences. 
Expression, purification, and identification of recombinant vimentin protein

The verified recombinant plasmid was transformed into E. coli BL21(DE3) competent cells (Tiangen) and cultured in a shaker of $37^{\circ} \mathrm{C}$ and $180 \mathrm{rpm}$. When the $\mathrm{OD}_{600}$ reached about 0.6. IPTG was added (final concentration $1 \mathrm{mmol} / \mathrm{l}$; Sigma-Aldrich, St. Louis, MO, USA) to induce and express recombinant vimentin protein. The cell pellet was collected by centrifugation and lysed with sonication at $4{ }^{\circ} \mathrm{C}$. Then the lysed product was analyzed by $10 \%$ sodium dodecyl sulfate-polyacrylamide gel electrophoresis (SDS-PAGE) to confirm that the recombinant protein was present as a soluble protein or in inclusion bodies. The recombinant protein was purified according to the characteristics using a published gel purification method [18]. Purified recombinant vimentin protein $(10 \mu \mathrm{g})$ was analyzed by SDS-PAGE and transferred to polyvinylidene fluoride (PVDF) membrane (Millipore, Billerica, MA, USA). The PVDF membrane was blocked in $5 \%$ skim milk for $2 \mathrm{~h}$ at $37^{\circ} \mathrm{C}$ and incubated with AntiGST Tag Mouse Monoclonal Antibody (1:2000; CWBio, Beijing, China) for $2 \mathrm{~h}$ at $37^{\circ} \mathrm{C}$, and was then washed in phosphate-buffered saline (PBS) three times for $5 \mathrm{~min}$ and incubated with peroxidase-conjugated AffiniPure Goat Anti-Mouse IgG (H+L) (1:5000; Proteintech, Chicago, IL, USA) for $45 \mathrm{~min}$ at room temperature. Finally, imaging was obtained using the ChemiDoc Touch Imaging System (Bio-Rad, Hercules, CA, USA).

\section{Collection of DF-1 cells infected or not infected with $E$. tenella sporozoites}

DF-1 cells $\left(1.5 \times 10^{6}\right.$ cells/well $)$ were plated into six-well plates and cultured at $37{ }^{\circ} \mathrm{C}$ in a $5 \% \mathrm{CO}_{2}$ incubator to a cell coverage of $80-90 \%$. Freshly isolated sporozoites were incubated with DMEM (2\% FBS, 5\% PBS) for $2 \mathrm{~h}$ at $37^{\circ} \mathrm{C}$. The DF-1 cells were infected with pretreated sporozoites with a multiplicity of infection of $3(\mathrm{MOI}=3)$. At different time points during infection $(1,2,6,12,24,36,48$, 60 , and $72 \mathrm{~h}$ ), the medium and noninvasive sporozoites were removed by washing gently with PBS three times. The DF-1 cells without infecting sporozoites were set as the uninfected control. Every cell sample was harvested with a cell scraper and stored at $-80{ }^{\circ} \mathrm{C}$ for subsequent analysis.

\section{Assessment of vimentin mRNA transcription levels after sporozoite infection}

Total RNA was extracted from cells collected from different time points using the MiniBEST Universal RNA Extraction Kit (TaKaRa) according to the manufacturer's instructions. The design of specific primers was based on published vimentin and GAPDH gene sequences, as follows: vimentin, forward primer: 5'-GCAAAGTTGAGT
CCCTGCAA- $3^{\prime}$, reverse primer: $5^{\prime}$-AGGGCAGCAGTA AGATCAGG-3'; GAPDH, forward primer: $5^{\prime}$-GGCACT GTCAAGGCTGAGAACG-3', reverse primer: $5^{\prime}$ - TGA GATGATAACACGCTTAGCACCAC- $3^{\prime}$. Vimentin mRNA transcription levels in each group of samples were determined using the One-Step TB Green PrimeScript RT-PCR Kit II (TaKaRa), according to the manufacturer's instructions. Each reaction was carried out in triplicate, and the experiment was performed three times. The relative expression of vimentin mRNA was calculated using the $2^{-\Delta \Delta C t}$ method [19], and SPSS 22.0 (https://www.ibm. com) was used for t-test analysis.

\section{Analysis of vimentin expression levels after sporozoite infection}

Western blotting was used to detect vimentin expression levels during sporozoite infection. Proteins at different time points $(6,36$, and $72 \mathrm{~h})$ were extracted from infected and uninfected samples using cell lysis buffer IP (Beyotime, Haimen, China) for western blotting. Protein concentration was determined by a BCA Protein Assay Kit (Beyotime), then $10 \mu \mathrm{g}$ of protein was taken from each group for SDS-PAGE and transferred to PVDF membrane. The PVDF membrane was blocked in 5\% skim milk for $2 \mathrm{~h}$ at $37^{\circ} \mathrm{C}$ and then incubated with polyclonal rabbit antibody against vimentin (1:200, prepared by our laboratory) or $\beta$-GAPDH (internal reference, 1:2000; Yeasen, Shanghai, China) for $2 \mathrm{~h}$ at $37{ }^{\circ} \mathrm{C}$. They were then washed in PBS three times for $5 \mathrm{~min}$ and incubated with goat anti-rabbit IgG antibody (1:5000; LI-COR Biosciences, Lincoln, NE, USA) for $45 \mathrm{~min}$ at room temperature. Finally, images were obtained with the ChemiDoc Touch Imaging System.

\section{Determination of vimentin phosphorylation levels after sporozoite infection}

Proteins were extracted from infected and uninfected cells collected from different time points $(1,2,6,12$, $24,36,48,60$, and $72 \mathrm{~h}$ ) using a phosphorylated protein extraction kit (Solarbio, Beijing, China). Protein concentration was determined using a BCA protein assay kit (Beyotime), and $10 \mu \mathrm{g}$ of protein from each group was used for Phos-tag acrylamide-based gel (Wako, Tokyo, Japan) analysis. After electrophoresis, Phos-tag acrylamide gels were immersed three times in transfer buffer containing $1 \mathrm{mM}$ EDTA for $10 \mathrm{~min}$ and then washed in transfer buffer without EDTA for $10 \mathrm{~min}$. Then the proteins were transferred to a PVDF membrane for western blot analysis. 
Table 1 Sequences of siRNA used to downregulate the vimentin expression

\begin{tabular}{ll}
\hline siRNA no. & siRNA sequences \\
\hline siRNA-1 & 5'-CCAUCAACACGGAGUUCAATT-3' \\
siRNA-2 & 5'-CCGACAGGAUGUUGACAAUTT-3' \\
siRNA-3 & 5'-GGAAGAAAUGGCUCGCCAUTT-3' \\
Negative siRNA & 5'-UUCUCCGAACGUGUCACGUTT-3' \\
\hline
\end{tabular}

\section{Assessment of vimentin distribution after sporozoite infection}

Cell climbing films were placed in six-well plates. DF- 1 cells $\left(1.5 \times 10^{6}\right.$ cells/well $)$ were plated into the six-well plates and cultured to a cell coverage of 80\%-90\%. Sporozoites were labeled with PBS containing carboxyfluorescein diacetate succinimidyl ester (CFSE) (1:20,000; Invitrogen, Carlsbad, CA, USA) for 15 min and then incubated with DMEM (2\% FBS, 5\% PS) for $2 \mathrm{~h}$ at $37{ }^{\circ} \mathrm{C}$. Labeled sporozoites were added to the DF-1 cells with a multiplicity of infection of 3 $(\mathrm{MOI}=3)$. Samples were collected from the six-well culture plates at different time points $(1,12$, and $24 \mathrm{~h}$ ). The uninfected and infected DF-1 cells were fixed with 4\% paraformaldehyde (Solarbio) for $15 \mathrm{~min}$, permeabilized with $0.1 \%$ Triton $\mathrm{X}-100$ for $15 \mathrm{~min}$, and blocked in $2 \%$ bovine serum albumin (BSA) (Solarbio) at $4{ }^{\circ} \mathrm{C}$ for $12 \mathrm{~h}$. DF-1 cells were incubated with polyclonal rabbit antibody against vimentin $(1: 200)$ for $2 \mathrm{~h}$ at $37{ }^{\circ} \mathrm{C}$, and then washed in PBS three times for $5 \mathrm{~min}$ and incubated with Alexa Fluor 647 Goat Anti-Rabbit IgG (1:500; Invitrogen) for $45 \mathrm{~min}$ at room temperature. DF-1 cells were washed again with PBS, stained with DAPI (1:500; Beyotime) for 15 min, treated with Fluoromount Aqueous Mounting Medium (Sigma-Aldrich), and imaged under a fluorescence microscope (Olympus, Tokyo, Japan).

\section{Analysis of anti-vimentin polyclonal antibodies on sporozoite invasion}

DF-1 cells $\left(3.0 \times 10^{5}\right.$ cells/well $)$ were plated into 24 -well plates and cultured to a cell coverage of $80-90 \%$. Polyclonal rabbit antibody against vimentin (prepared by our laboratory) was purified using protein $A+G$ agarose (Beyotime). Then, purified IgG was added to DF-1 cells at a final concentration of 25, 50, 100, 200, 300, or $400 \mu \mathrm{g} / \mathrm{ml}$ in DMEM (2\% FBS, 5\% PS) at $37{ }^{\circ} \mathrm{C}$ for $3 \mathrm{~h}$, respectively. The same quantity of purified IgG from rabbit sera was used as negative control, and DF-1 cells incubated without antibodies were used as a positive control. After $3 \mathrm{~h}$, the original medium was replaced with DMEM (2\% FBS, 5\% PS), and the sporozoite invasion rate was determined by an invasion assay in vitro [9]. Labeled sporozoites (as described above) were added to DF-1 cells at a multiplicity of infection of $3(\mathrm{MOI}=3)$. After invasion for $6 \mathrm{~h}$ at $37^{\circ} \mathrm{C}$ in $5 \% \mathrm{CO}_{2}$, DF-1 cells were washed with PBS to remove noninvasive sporozoites, digested with trypsin, and analyzed by flow cytometry (Cytomics FC 500; Beckman Coulter, USA). A total of three tests were carried out.

\section{Effects of downregulation of vimentin expression by small interfering RNA (siRNA) on sporozoite invasion ability} Transfection experiments were performed when cell coverage reached $40-50 \%$ using the siRNA sequences published by Schäfer et al. (Table 1) [20]. The DF-1 cells were transfected with siRNA $1+2+3$ or negative siRNA using Lipofectamine 3000 (Invitrogen) according to the manufacturer's instructions. Briefly, DNA and the transfection reagent were mixed (10 ml lipofectamine 3000 and $4 \mu \mathrm{g}$ DNA), incubated at room temperature for $30 \mathrm{~min}$, and added to the cells. Six hours later, the DNA-transfection reagent mixture was replaced by DMEM containing $10 \%$ FBS. All plasmids were transfected in triplicates. In the mock-treated cells, only the transfection reagent was used. At $24 \mathrm{~h}$ post-transfection, the protein samples were collected to test vimentin expression levels by western blot analysis.

In order to determine the cell growth after transfection with siRNAs, we examined cell activity. DF-1 cells $\left(0.8 \times 10^{5}\right.$ cells $)$ were plated into 96 -well plates, then transfected using the above method. The original medium was discarded after $24 \mathrm{~h}$ transfection, $90 \mu \mathrm{l}$ complete medium and $10 \mu \mathrm{l}$ CCK- 8 solution were added

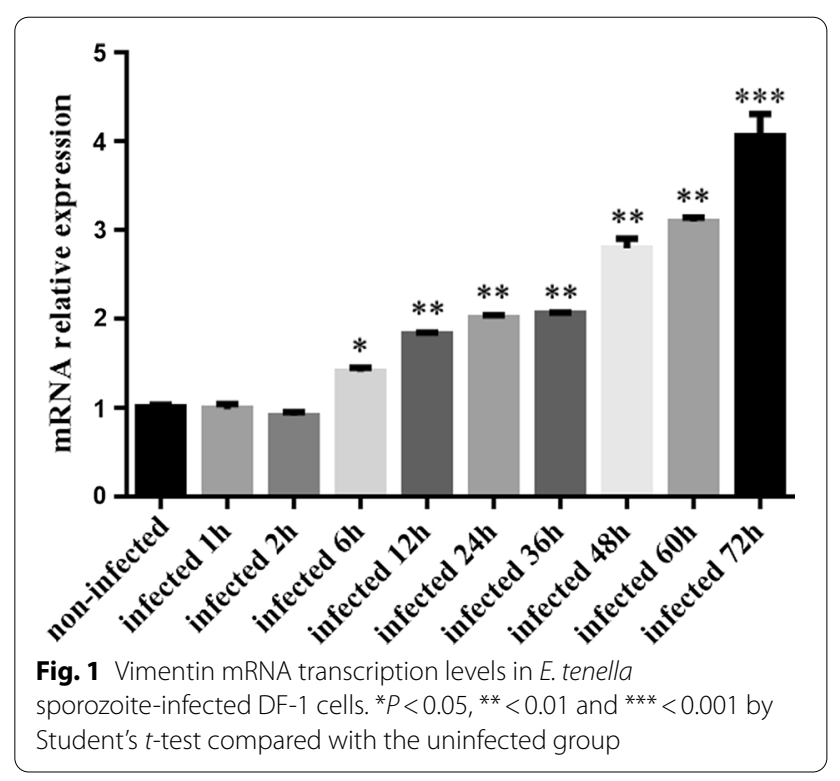


to each well, and then the $\mathrm{OD}_{450}$ value of the cells was measured every hour for $6 \mathrm{~h}$.

DF-1 cells $\left(3.0 \times 10^{5}\right.$ cells $)$ were plated into 24-well plates and transfected with siRNA. After $24 \mathrm{~h}$ transfection, the CFSE-labeled sporozoites (as described above) were added to DF-1 cells at a multiplicity of infection of $3(\mathrm{MOI}=3)$. After invasion for $6 \mathrm{~h}$ at $37^{\circ} \mathrm{C}$ in $5 \% \mathrm{CO} 2$, DF-1 cells were washed with PBS to remove noninvasive sporozoites, digested with trypsin, and analyzed by flow cytometry (Cytomics FC 500; Beckman Coulter, USA). DF-1 cells that were not transfected and transfected with negative siRNA were used as control groups. A total of three tests were carried out.

\section{Statistical analysis}

The experimental data were compared using SPSS version 22.0 statistical software (SPSS IBM Corp., Armonk, NY, USA). The $t$-test was used for two independent samples, and one-way analysis of variance (ANOVA) and Duncan's multiple range test were used for multiple groups. Significance was set at $P<0.05$, with $P<0.01$ indicating an extremely significant difference. GraphPad Prism 5 (GraphPad Software Inc., San Diego, CA, USA) was used for graphing the results.

\section{Results}

\section{Expression, purification, and validation of recombinant} vimentin protein

A band of about 1383 bp was obtained by polymerase chain reaction (PCR) amplification. A BLASTN comparison indicated that the obtained sequence was $99.9 \%$ homologous with the published G. gallus vimentin gene (GenBank accession number: NM_001048076.2). The

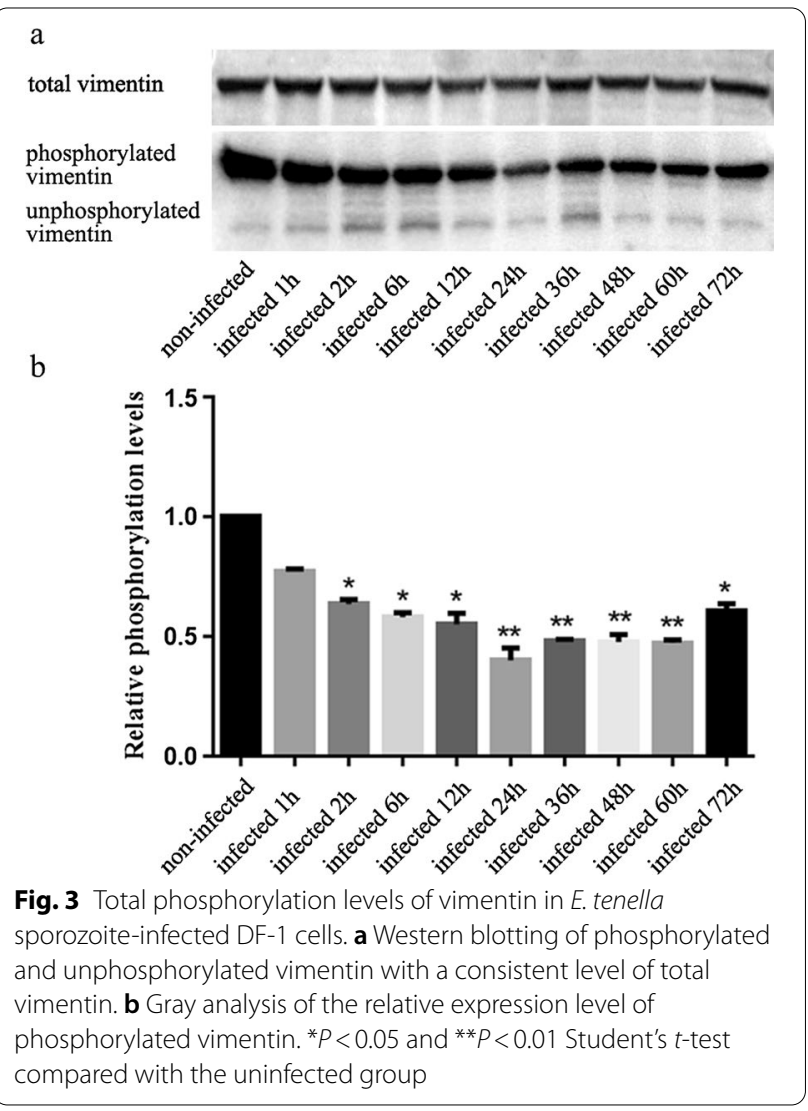

vimentin gene encoded a 460 aa protein with a molecular mass of about $53.2 \mathrm{kDa}$. A protein band of about $80 \mathrm{kDa}$ was observed in SDS-PAGE because a $26 \mathrm{kDa}$ GST tag was derived from the vector. The recombinant vimentin protein was purified by gel purification and recognized
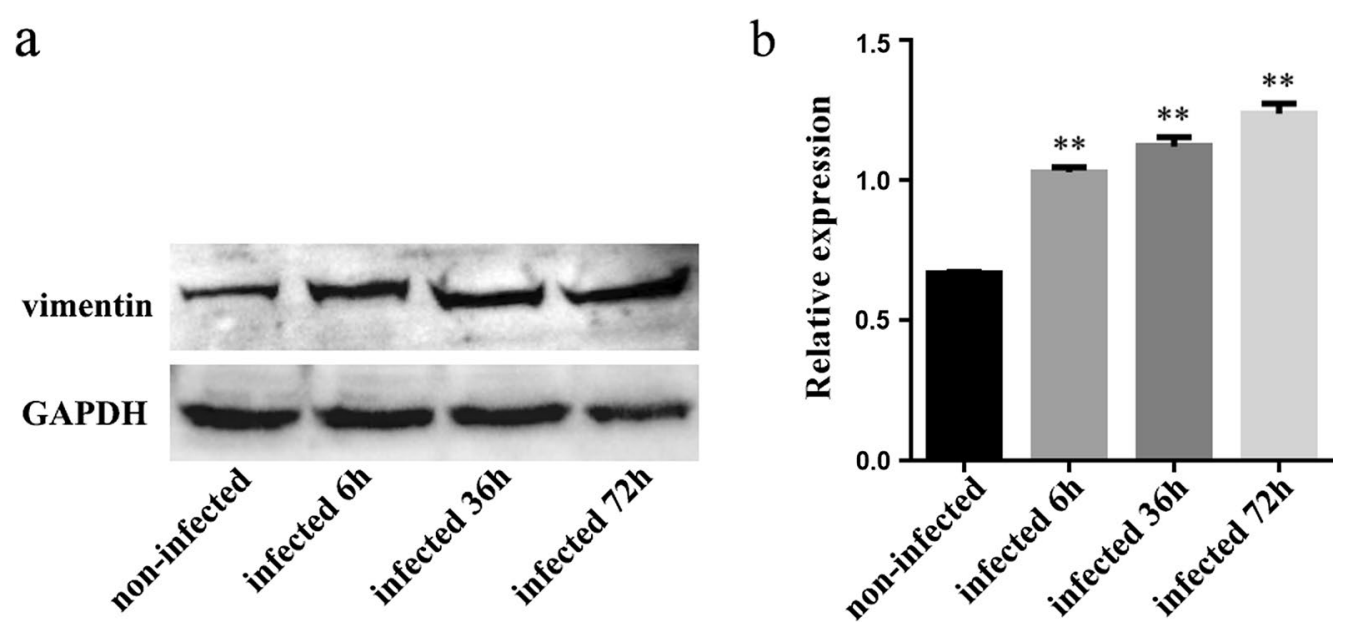

Fig. 2 Vimentin protein expression levels in E. tenella sporozoite-infected DF-1 cells. a Western blotting of the internal reference GAPDH and vimentin protein. $\mathbf{b}$ Gray analysis of the relative expression level of the vimentin protein. ${ }^{* *} P<0.01$ by Student's $t$-test compared with the uninfected group 


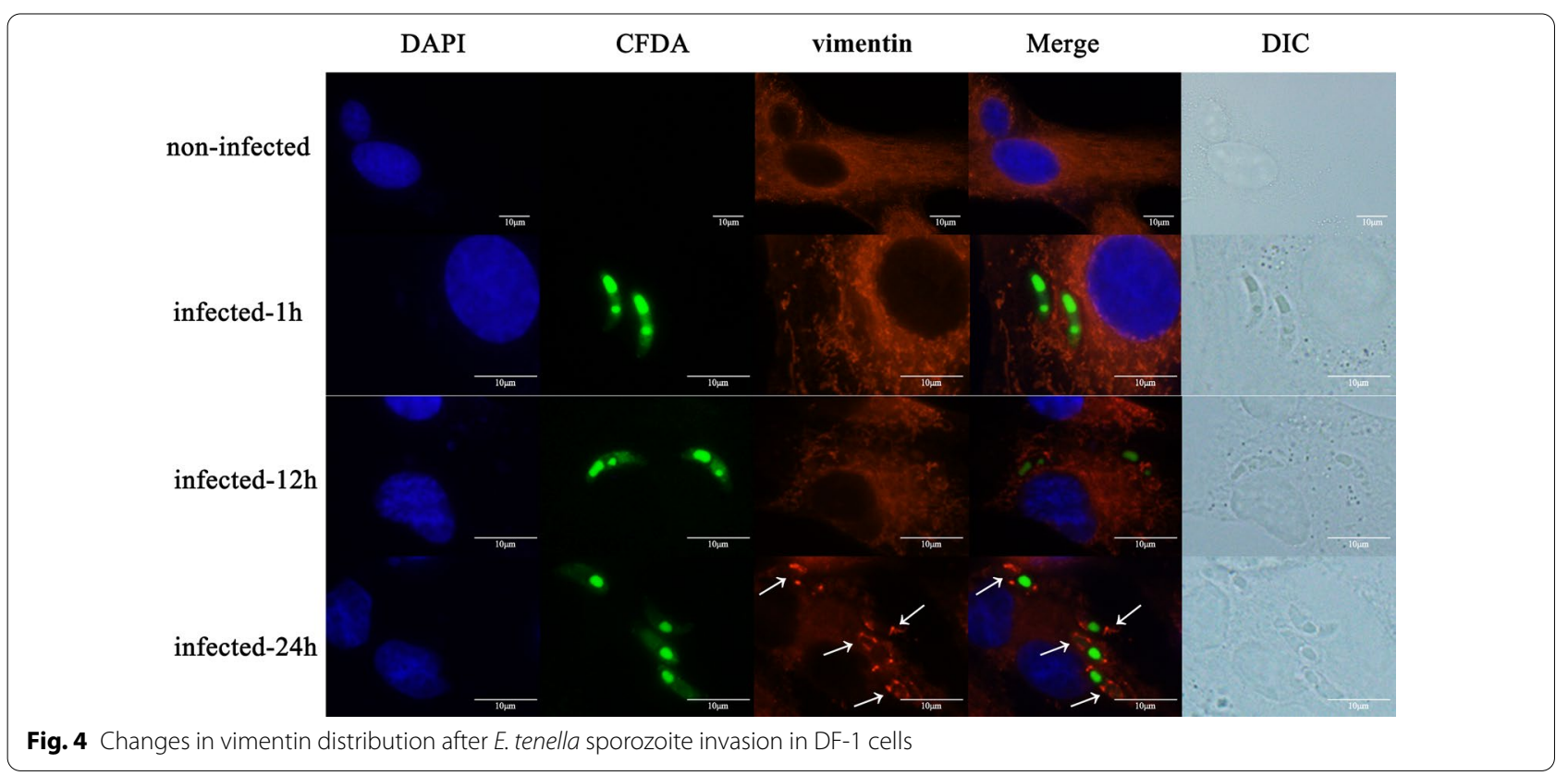

by anti-GST tag mouse monoclonal antibody (Additional file 1: Figure S1).

\section{Vimentin mRNA transcription levels after E. tenella sporozoite infection}

The mRNA levels of vimentin in DF-1 cells infected for 1 , 2, 6, 12, 24, 36, 48, 60, and $72 \mathrm{~h}$ and uninfected E. tenella sporozoites were measured using real-time quantity PCR (qPCR). The results showed that the transcriptional level of vimentin increased significantly $6-72 \mathrm{~h}$ after sporozoite infection (Fig. 1) $\left(F_{(7,16)}=244.681, P<0.0001\right)$. As compared with the uninfected group, the transcriptional levels of vimentin in the $1 \mathrm{~h}$ and $2 \mathrm{~h}$ infected groups were not significant $\left(F_{(2,6)}=2.625, P=0.152\right)$, that of in the $6 \mathrm{~h}$ infected group was higher $\left(F_{(1,4)}=13.500, P=0.021\right)$, and those of in 12-72 $\mathrm{h}$ infected groups increased significantly $\left(F_{(6,14)}=241.864, P<0.0001\right)$.

\section{Vimentin expression levels after E. tenella sporozoite infection}

The protein expression levels of vimentin in DF-1 cells infected for 6, 36, and $72 \mathrm{~h}$ and uninfected E. tenella sporozoites were measured using western blotting. The results showed that the protein expression levels of

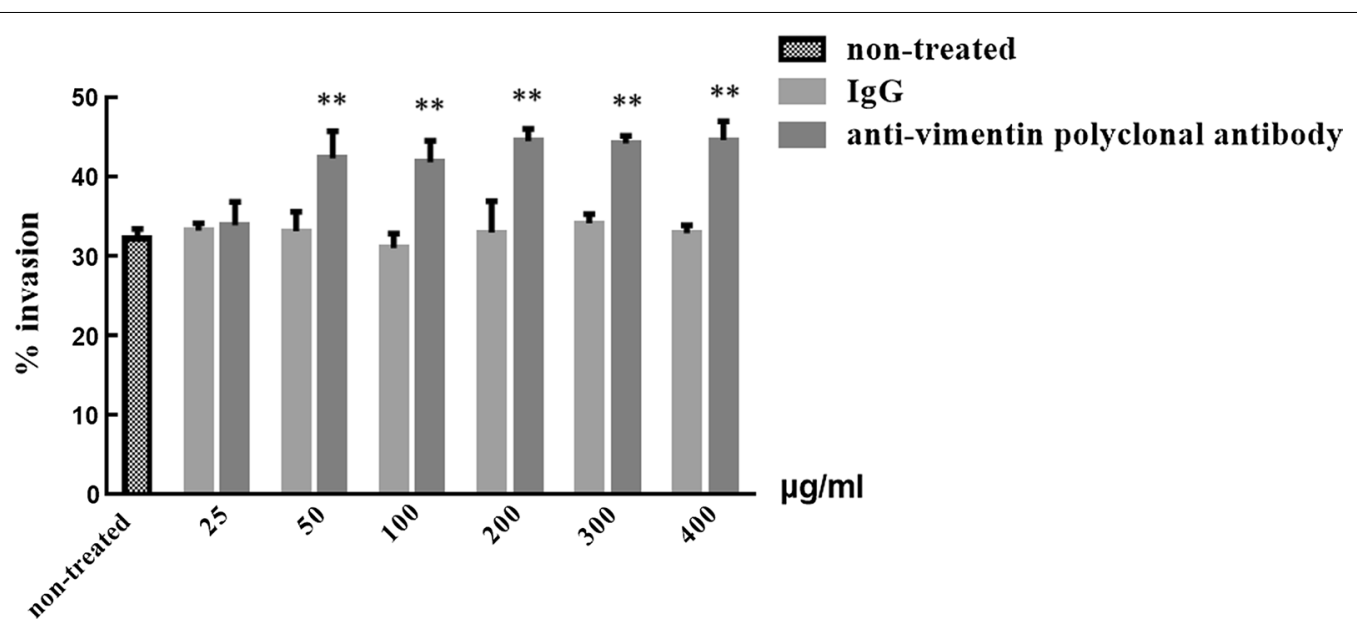

Fig. 5 Effect of anti-vimentin polyclonal antibody on E. tenella sporozoite invasion in DF-1 cells. ${ }^{*} P<0.01$ by Student's $t$-test compared with anti-vimentin polyclonal antibody treatment or with the same dose of normal rabbit lgG 
a

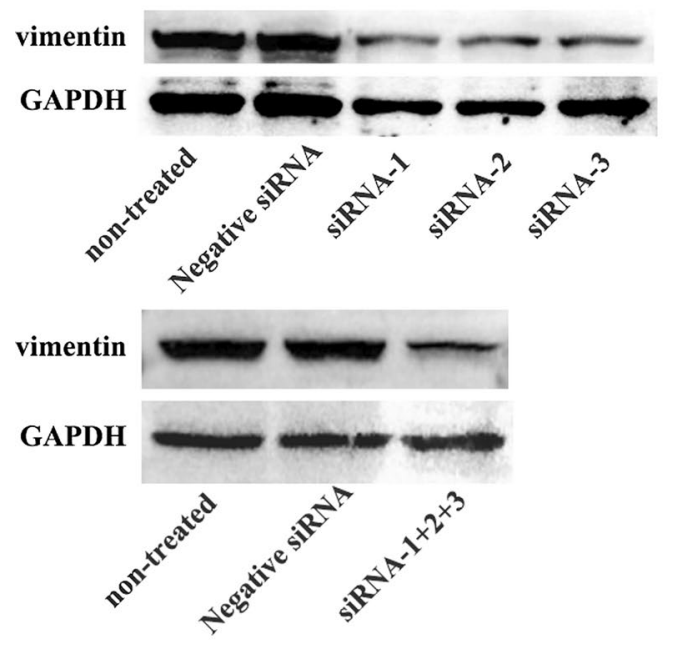

C

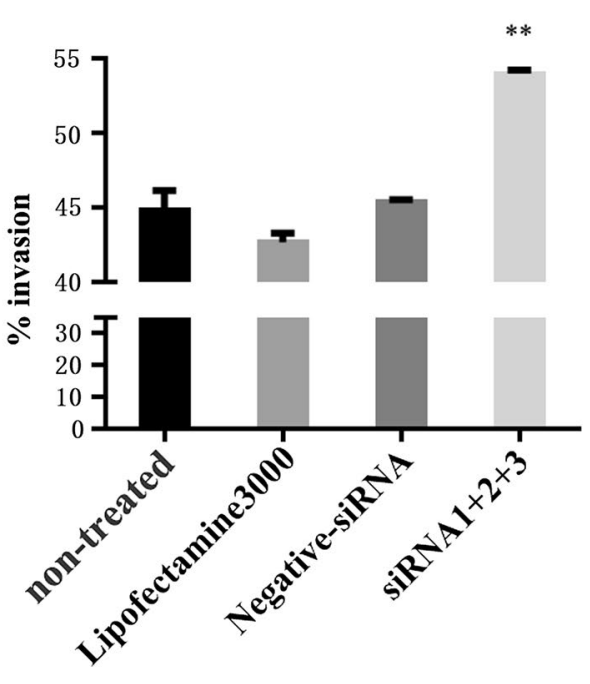

b

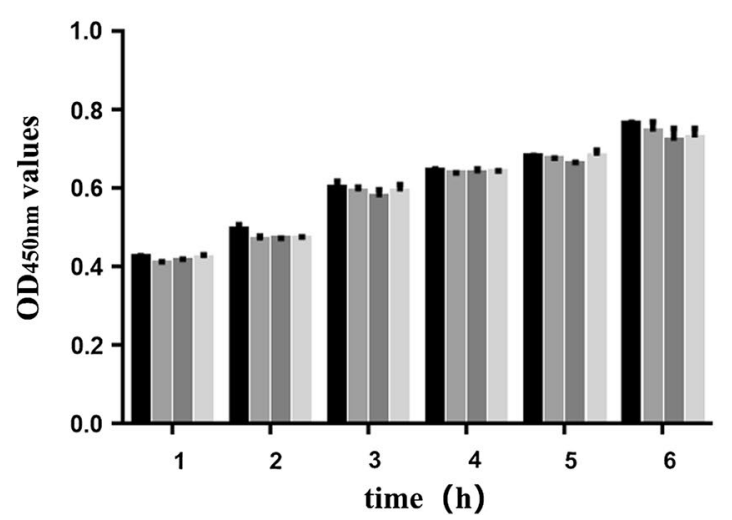

non-treated

Lipofectamine 3000

Negative-siRNA

SiRNA1+2+3

time (h)

Fig. 6 Effects of vimentin downregulation on sporozoite invasion in DF-1 cells. a The expression of vimentin protein in different transfection groups. b Cell growth curves of different transfection groups. $\mathbf{c}$ The invasion rate of sporozoites after downregulation of vimentin. ${ }^{*} P<0.01$ by Student's t-test compared with downregulated vimentin expression level or the untreated group

vimentin increased significantly $6-72 \mathrm{~h}$ after sporozoite infection (Fig. 2) $\left(F_{(3,8)}=20.204, P<0.0001\right)$.

\section{Total phosphorylation levels of vimentin after E. tenella sporozoite infection}

Total phosphorylation levels of vimentin in DF-1 cells infected for $1,2,6,12,24,36,48,60$, and $72 \mathrm{~h}$ and uninfected E. tenella sporozoites were measured using western blotting. The results showed that the total phosphorylation levels of vimentin in DF-1 cells decreased within $24 \mathrm{~h}$ of infection and reached the lowest level at
$24 \mathrm{~h}$ of infection, and then slightly increased (Fig. 3) $\left(F_{(9,20)}=16.362, P<0.0001\right)$.

\section{Vimentin distribution in DF-1 cells infected by $E$. tenella sporozoites}

The distribution of vimentin in DF-1 cells infected with $E$. tenella sporozoites for 1,12 , and $24 \mathrm{~h}$ and in uninfected DF-1 cells was determined using indirect immunofluorescence. The results showed that vimentin aggregated around the sporozoites $24 \mathrm{~h}$ after sporozoite infection (Fig. 4). 
Effect of rabbit anti-vimentin polyclonal antibodies on $E$. tenella sporozoite invasion of DF-1 cells

The sporozoite invasion rate increased significantly after treatment with rabbit anti-vimentin polyclonal antibodies compared with the same dose of normal rabbit IgG-treated and untreated DF-1 cells $\left(F_{(6,14)}=62.015\right.$, $P<0.0001)$. As compared with the same dose of naive rabbit sera IgG (used as negative controls), pretreatment at concentrations of $50-400 \mu \mathrm{g} / \mathrm{mL}$ significantly affected the invasion capacity of sporozoites. However, increasing the rabbit anti-vimentin polyclonal antibody concentration produced no significant change in the sporozoite invasion rate $\left(F_{(4,10)}=2.050, P=0.163\right)$, indicating that $50 \mu \mathrm{g} / \mathrm{ml}$ concentration of antibody could achieve a good promotion effect (Fig. 5).

Effects of downregulating vimentin expression on E. tenella sporozoite invasion of DF-1 cells

Western blot analysis of the interference effect of three siRNAs found that siRNA-1, siRNA-2, and siRNA-3 produced better interference effects than the untreated and negative siRNA control groups, and significantly downregulated vimentin protein expression $24 \mathrm{~h}$ after transfection (Fig. 6a). To determine the effect of downregulated vimentin expression level on cell activity and sporozoite invasion rate, DF-1 cells were transfected with siRNA-1, siRNA-2, and siRNA-3. The results showed that compared with the untreated group, treatment with siRNA-1+2+3, Lipofectamine 3000 , or negative siRNA had no significant effect on cell activity (Fig. 6b) $(P>0.05)$, but the invasion rate of sporozoites in the siRNA-1+2+3-treated group increased significantly $\left(F_{(1,4)}=174.781, P<0.0001\right)$, and the increase in Lipofectamine 3000-treated and negative siRNA-treated groups was not significant (Fig. 6c) $\left(F_{(2,6)}=1.494, P=0.298\right)$.

\section{Discussion}

Vimentin is a type III intermediate filament protein that is highly evolutionarily conserved in vertebrates and has no homologous proteins in coccidia. Vimentin, together with microtubules and microfilaments, constructs the network structure of protein fibers in cells, which is essential for the maintenance of cell morphology, intracellular material transport, and cell migration [21]. Besides these complex biological functions, vimentin is expressed on the cell surface and acts as a receptor to interact with the proteins of pathogens, regulating the pathogen infection process in host cells. For instance, some viruses and bacteria can bind to vimentin on the cell surface through specific virulence proteins or adhesion factors to facilitate their invasion of host cells [22, 23].
We used qPCR and western blotting to detect the mRNA transcriptional and protein expression levels of vimentin after $E$. tenella sporozoite invasion. The results showed that the transcription and expression levels of vimentin increased significantly in DF-1 cells 6-72 h after infection with sporozoites. Studies have shown that reticuloendotheliosis virus (REV) infection leads to upregulation of vimentin expression in chick embryo fibroblasts (CEF) [20], and Mycobacterium tuberculosis infection causes the downregulation of vimentin expression levels in macrophages [24]. In CoS 7 cells infected with Toxoplasma gondii, the expression levels of vimentin were dynamic, first showing a decreasing trend, then an increase and subsequent decrease, and finally an increase [14]. The changes in vimentin expression levels differed among different pathogen infections, which suggests that vimentin may play different roles in different pathogen infections.

We used indirect immunofluorescence to detect the distribution of vimentin in the process of sporozoite infection. The results showed that after $24 \mathrm{~h}$ of infection, the vimentin accumulated around the sporozoites in DF-1 cells, which was similar to results found in other pathogens. Vimentin rearrangements occurred around parasitophorous vacuole membrane (PVM) after $1 \mathrm{~h}$ of T. gondii invasion of host cells [25]. Early in African swine fever virus (ASF) and hantavirus infection, vimentin showed stellate aggregation on one side of the Vero cells and then rearranged into a cage structure around the virus replicator [26, 27]. Based on our results, combined with those of existing studies, we speculate that this aggregation may be a protective mechanism for host cells against the spread of pathogens in the cytoplasm. However, the role of vimentin rearrangement in pathogen infection is unclear.

We found that the total phosphorylation level of vimentin changed dynamically in DF-1 cells within $72 \mathrm{~h}$ of sporozoite infection. Our previous phosphorylation proteomics results also confirmed that phosphorylation levels at multiple sites in vimentin changed after sporozoite infection. Previous studies have found that the infection process of many Apicomplexa phylum parasites was accompanied by the rearrangement of the host cytoskeleton, and this dynamic change in the cytoskeleton was closely related to the parasite invasion efficiency [7, 2832]. The change in vimentin distribution is influenced by solubility, which is regulated by phosphorylation [33, 34]. Therefore, we speculated that sporozoites may affect the host cytoskeleton structure by regulating the phosphorylation level of vimentin, creating favorable conditions for invasion and intracellular parasitism.

To further investigate the role of vimentin in DF-1 cells infected with sporozoites, we used an invasion 
assay in vitro to observe the effect of vimentin polyclonal antibodies or downregulating the expression of vimentin in DF-1 cells on sporozoite invasion. We found that both treatments significantly promoted sporozoite invasion, indicating that vimentin may inhibit E. tenella sporozoite invasion. Our results were consistent with those from $T$. gondii, but different from those for bacteria and viruses. Studies have shown that vimentin antibodies inhibited the Listeria monocytogenes invasion of human cerebral microvascular endothelial cells (hCMEC) and the survival of M. tuberculosis in macrophages [24, 35]. The knockout of vimentin can promote the invasion of $T$. gondii in hCMEC but has no significant effect on intracellular proliferation and release [14]. Silencing or destroying the vimentin protein structure can inhibit the replication and proliferation of avian reticuloendothelial proliferative virus in CEF cells [20], while for dengue virus type 2, vimentin knockout can promote the invasion of hCMEC [36]. These results suggest that vimentin plays different roles in the process of infection of different pathogens. After invasion, E. tenella sporozoites round up into trophozoites at about $36 \mathrm{~h}$ post-infection and undergo schizogony (asexual multiple fission) at $48-72 \mathrm{~h}$ post-infection, resulting in the production of multiple first-generation merozoites. Both mRNA transcriptional and protein expression levels of vimentin increased significantly during these periods, but its specific role in the intracellular development of $E$. tenella sporozoites needs further study.

To sum up, E. tenella sporozoite invasion caused changes in host vimentin distribution, and vimentin inhibited the sporozoite invasion of DF-1 cells. However, the specific role of vimentin in coccidian infection remains to be further investigated.

\section{Abbreviations}

NCBI: National Center for Biotechnology Information; BLAST: Basic Local Alignment Search Tool; MOI: Multiplicity of infection.

\section{Supplementary Information}

The online version contains supplementary material available at https://doi. org/10.1186/s13071-021-05107-4.

Additional file 1: Figure S1. Expression, purification, and validation of vimentin recombinant protein. a Lane M: protein marker; Lane 1: negative control (not induced with IPTG); Lanes 2: induced with IPTG for 2 h. b Lane 1: purified vimentin detected by SDS-PAGE. $\mathbf{c}$ Lane 1: purified vimentin verified by western blotting.

\section{Authors' contributions}

HD conceived and designed the study. ZL and XFG performed the experiments. ZL and QPZ collected parasites. HD and ZL analyzed the data. ZL and $\mathrm{HYH}$ wrote the manuscript. All authors read and approved the final manuscript.

\section{Funding}

This work was supported by grants from the National Key R\&D Program of China (No. 2017YFD0500400) and the National Parasitic Resources Center (No. NPRC-2019-194-30).

\section{Availability of data and materials}

Not applicable.

\section{Declarations}

\section{Ethics approval and consent to participate}

All animal procedures were approved by the Animal Ethics Committee of Shanghai Veterinary Institute, Chinese Academy of Agricultural Science. Experiments were conducted in accordance with animal ethics guidelines and approved protocols.

\section{Consent for publication}

Not applicable.

\section{Competing interests}

The authors declare that they have no competing interests.

\section{Author details}

${ }^{1}$ Key Laboratory of Animal Parasitology of Ministry of Agriculture, Shanghai Veterinary Research Institute, Chinese Academy of Agricultural Sciences, Minhang 200241, Shanghai, People's Republic of China. ${ }^{2}$ Beijing YuanDa Spark Medicine Technology Co., Ltd, Beijing 100088, People's Republic of China.

Received: 15 July 2021 Accepted: 19 November 2021

Published online: 04 January 2022

References

1. Soutter F, Werling D, Tomley FM, Blake DP. Poultry coccidiosis: design and interpretation of vaccine studies. Front Vet Sci. 2020;7:101.

2. Chapman HD. Biochemical, genetic and applied aspects of drug resistance in Eimeria parasites of the fowl. Avian Pathol. 1997;26:221-44.

3. Sharman PA, Smith NC, Wallach MG, Katrib M. Chasing the golden egg: vaccination against poultry coccidiosis. Parasite Immunol. 2010;32:590-8

4. Del Cacho E, Gallego M, Lillehoj HS, Quilez J, Lillehoj EP, Sánchez-Acedo C. Induction of protective immunity against experimental Eimeria tenella infection using serum exosomes. Vet Parasitol. 2016;224:1-6.

5. Pelléruy L. Life cycles involving sexual and asexual generations of Eimeria in gallinaceous birds. Parasit Hung. 1970;44:133-46.

6. Lal K, Bromley E, Oakes R, Prieto JH, Sanderson SJ, Kurian D, et al. Proteomic comparison of four Eimeria tenella life-cycle stages: unsporulated oocyst, sporulated oocyst, sporozoite and second-generation merozoite. Proteomics. 2009;9:4566-76.

7. Wu C, Wang Z, Li J, Lin X, Liao S, Qi N, et al. A role for host cell F-actin polymerization during the protozoan parasite infection. Acta Veterinaria et Zootechnica Sinica. 2016:47:423-8 (in Chinese).

8. Tierney JB, Matthews E, Carrington SD, Mulcahy G. Interaction of Eimeria tenella with intestinal mucin in vitro. J Parasitol. 2007:93:634-8.

9. Wang L, Zhu S, Zhao Q, Huang B, Lv L, Liu G, et al. Effects of host fatty acid-binding protein 4 on Eimeria tenella sporozoites invasion of cells. Parasitol Res. 2019;118:1919-26.

10. Zhao Z, Zhu S, Huang B, Han H, Zhao Q, Lv L, et al. Preliminary study of RACK1 effects on Eimeria tenella sporozoites invasion of cells. Chin J Anim Infect Dis. 2018;26:62-8 (in Chinese).

11. Turkki P, Laajala M, Flodström-Tullberg M, Marjomäki V. Human enterovirus group $B$ viruses rely on vimentin dynamics for efficient processing of viral nonstructural proteins. J Virol. 2020;94:e01393-e1419.

12. Chen W, Gao N, Wang JL, Tian YP, Chen ZT, An J. Vimentin is required for dengue virus serotype 2 infection but microtubules are not necessary for this process. Arch Virol. 2008;153:1777-81.

13. Zou Y, He L, Huang SH. Identification of a surface protein on human brain microvascular endothelial cells as vimentin interacting with 
Escherichia coli invasion protein IbeA. Biochem Biophys Res Commun. 2006;351:625-30.

14. He C, Kong L, Zhou L, Xia J, Wei H, Liu M, et al. Host cell vimentin restrains Toxoplasma gondii invasion and phosphorylation of vimentin is partially regulated by interaction with TgROP18. Int J Biol Sci. 2017;13:1126-37.

15. Tomley F. Techniques for isolation and characterization of apical organelles from Eimeria tenella sporozoites. Methods. 1997;13:171-6.

16. Han $H Y$, Lin JJ, Zhao QP, Dong $H$, Jiang $L L$, $X u M Q$, et al. Identification of differentially expressed genes in early stages of Eimeria tenella by suppression subtractive hybridization and CDNA microarray. J Parasitol. 2010;96:95-102.

17. Xie MQ, Gilbert JM, Fuller AL, McDougald LR. A new method for purification of Eimeria tenella merozoites. Parasitol Res. 1990;76:566-9.

18. Burgess RR. Elution of proteins from gels. Methods Enzymol. 2009:463:565-72

19. Livak KJ, Schmittgen TD. Analysis of relative gene expression data using real-time quantitative PCR and the 2 (-Delta Delta $C(T)$ ) Method. Methods. 2001;25:402-8.

20. Schäfer G, Graham LM, Lang DM, Blumenthal MJ, Bergant Marušič M, et al. Vimentin modulates infectious internalization of human papillomavirus 16 pseudovirions. J Virol. 2017;91:e00307-e317.

21. Ivaska J, Pallari HM, Nevo J, Eriksson JE. Novel functions of vimentin in cell adhesion, migration, and signaling. Exp Cell Res. 2007:313:2050-62.

22. Patteson AE, Vahabikashi A, Goldman RD, Janmey PA. Mechanical and non-mechanical functions of filamentous and non-filamentous vimentin. Bioessays. 2020:42:e2000078.

23. Wang W, Sun J, Wang N, Sun Z, Ma Q, Li J, et al. Enterovirus A71 capsid protein VP1 increases blood-brain barrier permeability and virus receptor vimentin on the brain endothelial cells. J Neurovirol. 2020;26:84-94.

24. Mahesh PP, Retnakumar RJ, Mundayoor S. Downregulation of vimentin in macrophages infected with live Mycobacterium tuberculosis is mediated by reactive oxygen species. Sci Rep. 2016;6:21526.

25. Halonen SK, Weidner E. Overcoating of Toxoplasma parasitophorous vacuoles with host cell vimentin type intermediate filaments. J Eukaryot Microbiol. 1994;41:65-71.

26. Stefanovic S, Windsor M, Nagata KI, Inagaki M, Wileman T. Vimentin rearrangement during African swine fever virus infection involves retrograde transport along microtubules and phosphorylation of vimentin by calcium calmodulin kinase II. J Virol. 2005;79:11766-75.

27. Ramanathan HN, Chung DH, Plane SJ, Sztul E, Chu YK, Guttieri MC, et al. Dynein-dependent transport of the Hantaan virus nucleocapsid protein to the endoplasmic reticulum-Golgi intermediate compartment. J Virol. 2007;81:8634-47.

28. Hermosilla C, Schröpfer E, Stowasser M, Eckstein-Ludwig U, Behrendt $\mathrm{JH}$, Zahner H. Cytoskeletal changes in Eimeria bovis-infected host endothelial cells during first merogony. Vet Res Commun. 2008;32:521-31.

29. Na RH, Zhu GH, Luo JX, Meng XJ, Cui L, Peng HJ, et al. Enzymatically active Rho and Rac small-GTPases are involved in the establishment of the vacuolar membrane after Toxoplasma gondii invasion of host cells. BMC Microbiol. 2013;13:125.

30. Warncke JD, Beck HP. Host cytoskeleton remodeling throughout the blood stages of Plasmodium falciparum. Microbiol Mol Biol Rev. 2019:83:e00013-19.

31. Elliott DA, Coleman DJ, Lane MA, May RC, Machesky LM, Clark DP. Cryptosporidium parvum infection requires host cell actin polymerization. Infect Immun. 2001;69:5940-2.

32. Delorme-Walker V, Abrivard M, Lagal V, Anderson K, Perazzi A, Gonzalez V, et al. Toxofilin upregulates the host cortical actin cytoskeleton dynamics, facilitating Toxoplasma invasion. J Cell Sci. 2012;125:4333-42

33. Snider NT, Omary MB. Post-translational modifications of intermediate filament proteins: mechanisms and functions. Nat Rev Mol Cell Biol. 2014;15:163-77.

34. Eriksson JE, He T, Trejo-Skalli AV, Härmälä-Braskén AS, Hellman J, Chou YH, et al. Specific in vivo phosphorylation sites determine the assembly dynamics of vimentin intermediate filaments. J Cell Sci. 2004;117:919-32.
35. Ghosh P, Halvorsen EM, Ammendolia DA, Mor-Vaknin N, O'Riordan MXD, Brumell JH, et al. Invasion of the brain by Listeria monocytogenes is mediated by InIF and host cell vimentin. Bio. 2018;9:e00160-18.

36. Ramos I, Stamatakis K, Oeste CL, Pérez-Sala D. Vimentin as a multifaceted player and potential therapeutic target in viral infections. Int J Mol Sci. 2020;21:4675

\section{Publisher's Note}

Springer Nature remains neutral with regard to jurisdictional claims in published maps and institutional affiliations.
Ready to submit your research? Choose BMC and benefit from:

- fast, convenient online submission

- thorough peer review by experienced researchers in your field

- rapid publication on acceptance

- support for research data, including large and complex data types

- gold Open Access which fosters wider collaboration and increased citations

- maximum visibility for your research: over $100 \mathrm{M}$ website views per year

At BMC, research is always in progress.

Learn more biomedcentral.com/submissions 